\title{
Workspace Performance Investigation of a Multi-DOF Parallel Robot Manipulator
}

\author{
Ruixia Li and Fenxia Li
}

\begin{abstract}
A five degree of freedom (DOF) parallel robot manipulator is proposed in this paper. Kinematics, workspace and dexterity performance of the 5-SPS-UPU parallel robot manipulator were systematically deduced. The proposed mechanism with two rotations and three translations includes the fixed platform, five identical legs, a passive-constrained leg, the moving platform and some joints. First the inverse of kinematics and Jacobian matrix was derived by using the coordinate transformation theory and differentiation method. Second the workspace of the novel robot is analyzed by the boundary search method through the software. A numerical analysis of the variation of posture and position workspace of the robot is presented. Third the dexterity performance of the proposed manipulator has been analyzed, and the best kinematic dexterity is 1.1. The analytic results show that the robot mentioned in this paper has a large effective workspace and dexterity, which can be widely employed in the field of industrial robots.
\end{abstract}

Index Terms-Parallel manipulator, kinematics analysis, workspace, dexterity.

\section{INTRODUCTION}

As is known to all, integrated manufacturing technology highly relies on the development of higher performance robotic system. Compared with serial robots, the parallel manipulator has different potential advantages, including high stiffness, high accuracy and high loading capacities. However, the workspace of the parallel manipulator is relatively low. So it is necessary to research the workspace in the engineering application. Evaluation and optimization of the kinematics performance is the basic parallel robot structure design, driver selection and control mechanism. With the increasing application of parallel robot manipulator such as machine tools, simulators, cutting and welding machines as well as CNC machines has aroused general attention [1], [2]. At present, the optimization design of parallel manipulator are mainly concentrated on the workspace, dexterity, accuracy, stiffness and bearing capacity etc., the dexterous workspace are the most important kinematics performance index widely used to evaluate the kinematics accuracy. Recently, many scholars have studied on the five degrees of freedom parallel manipulators, such as Wang [3] analyzed the kinematics of a

Manuscript received August 1, 2018, revised October 21, 2018. This work was supported by the financial support of the Teaching Reform and Innovation Project for Shanxi Universities under Grant No.J2018136 and General Project of Changzhi Medical College under Grant QDZ201655.

Ruixia Li is with the Faculty of Biomedicine Engineering at Changzhi Medical College, Changzhi, Shanxi, China (e-mail: liruixiaczh@126.com).

Fenxia Li is with the Faculty of NC Engineering at Shanxi Institute of Mechanical and Electrical Engineering, Changzhi, Shanxi, China (e-mail:1fx5168@163.com). novel 4-SPS-CU parallel manipulator; Chen [4] studied the dexterity of the 4-UPS-UPU parallel manipulator by using the mean and variance, the global stiffness of the 5-UPS-CUU parallel was optimized by Zhang [5]. Kinematics performance on 5-DOF parallel manipulator 4SPS+RPS and 5SPS-UPU was analyzed and isotropic of the mechanism was designed by $\mathrm{Lu}$ [6]. Fu [7]studied the workspace of the novel three-leg six DOF parallel manipulator (TLPM) in detail, later they drawn the workspace and concluded that the robot has a large effective workspace with smooth boundary and can be widely applied in the field of industrial robots. Nowadays, most of the workspace research is concentrated on the robot's structure size and constraint conditions to search the workspace, which is achieved by the inverse solution of the kinematic equation in general [8].

Dexterity is one of the most important performances of parallel kinematic machines. Meanwhile, dimensional optimization is to improve the performance of a mechanical robotic system and is an important procedure of parallel manipulators from their schematic diagram to the structural design, in which two challenging issues associated with kinematic performance index and optimal design method are involved [9]. Therefore, it is quite necessary to perform the dexterity analysis and to conduct optimal design of the parallel kinematic machines in the early design stage. The determinant and condition number of Jacobian matrix have been widely used in performance evaluation and optimal design of parallel manipulators [10], [11]. Yet, Rezania et al. [12] pointed out that the unit inconsistent problem arises when these indices are applied to parallel manipulators with mixed DOFs of translations and rotations. Apart from the kinematic performance index associated with Jacobian matrix, some efforts were made to evaluate the motion or force transmissibility using screw theory [13]. Tsai [14] defined the virtual coefficient between the transmission wrench screw and the input velocity screw, while $\mathrm{Wu}$ et.al [15] proposed the input or output transmission index and dealt with kinematic evaluations of a 3-DOF parallel manipulator successfully.

In what follows, a new parallel manipulator that can generate three translations degrees of freedom and two rotations degrees of freedom is constructed. Its kinematic model and mathematical model are established and the Jacobian matrix is derived by the differential method. The workspace as one of the most important index of the parallel manipulator is computed considering the link length and angle constraints on the basis of the inverse solution of the kinematics. At last, the workspace of different orientation was drawn through the software MATLAB. We focus on the submatrix of the Jacobian matrix and adopt the dexterity performance index to solve the problem of units 
non-homogeneous.

\section{KINEMATICS ANALYSIS}

\section{A. Structure of the Parallel Manipulator}

In this work, the proposed 5-DOF parallel robot manipulator, illustrated Fig.1, is composed of five variable active legs (SPS) with identical topology and one constraint passive leg (UPU) which connect the base to the moving platform [16]. The 5SPS-UPU mechanism interconnection between the moving platform and the fixed base, which $S$ stands for spherical joint and $\mathrm{U}$ stands for Hooke joint, and $\mathrm{P}$ for the prismatic pair which is actuated independently. The middle leg is used to constrain the motion of the moving platform to only five degrees of freedom. The points pairs attached to the moving platform and the fixed platform are denoted by $A_{1}, A_{2}, A_{3}, A_{4}, A_{5}$ and $B_{1}, B_{2}, B_{3}, B_{4}, B_{5}$, respectively, link lengths are represented by $l_{1}, l_{2}, l_{3}, l_{4}, l_{5}$.

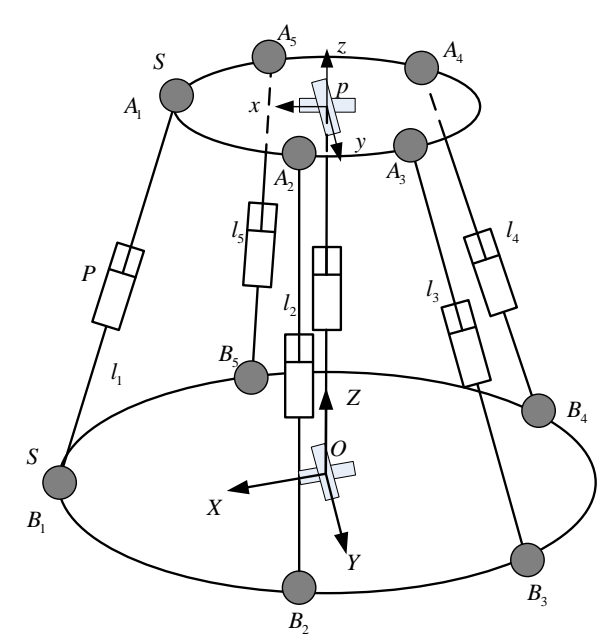

Fig. 1. The schematic of 5SPS-UPU parallel manipulator.

\section{B. Freedom Analysis and Calculation}

The structure of 5SPS-UPU parallel manipulator is shown in Fig.1. In order to enumerate the possibility, the Chebychev-Grübler-Kutzbach criterion is applied.A preliminary evaluation of the mobility of a kinematic chain can be found from the Chebychev-Grübler-Kutzbach formula [17].

$$
F=\mathrm{d}(n-g-1)+\sum_{i=1}^{g} f_{i}+v
$$

where,

$F:$ Spatial mechanism freedom;

$n$ : The number of parts

$g$ : The number of kinemics pairs

$f_{i}:$ Freedom of each pair

$v:$ The local freedom of mechanism

In this mechanism, the influence of the local freedom was eliminated due to the replacement of two spherical joints, which was completely equivalent with the branch UPS. According to the calculation formula, $d=6, n=14, g=18, \sum_{i=1}^{g} f_{i}=40, v=5$

So the freedom $F$ of the mechanism is equal to 5 , that is, three transitional motion along $X, Y$ and $Z$ axes, and two orientations about $\mathrm{X}$ and $\mathrm{Y}$ axes, i.e., roll $\psi$, pitch $\theta$, respectively.

\section{Inverse kinematics and Jacobian Analysis}

The kinematic structure of the proposed 5-DOF parallel robotic manipulator as shown in Fig.1. Suppose that the center of the $\mathrm{S}$ joint located on the base and on the platform are located on the circles with radius $r_{a}$ and $r_{b}$ respectively. A fixed coordinated frame $O-X Y Z$ is attached to the base center $O$ and a moving coordinate frame $p-x y z$ is attached on the platform. $x$ axis point to $A_{1}$ and $X$ axis point to the $B_{1}$. Spherical joints were uniformly distributed.

The position vectors of $A_{i}$ points are expressed in the moving coordinate frame as the following:

$$
{ }^{p} A_{i}=r_{a}\left[\begin{array}{lll}
c \alpha_{i} & s \alpha_{i} & 0
\end{array}\right]^{T}, \alpha_{i}=\frac{(i-1) 2 \pi}{5}, i=1,2,3,4,5
$$

where, $\mathrm{s}$ and $\mathrm{c}$ present sine and cosine, respectively, $\alpha$ represents the angle between $O A_{i}$ and the positive direction of $x$ axis,

Similarly, the position vectors of points $B_{i}$ are expressed in the reference frame as the following:

$$
{ }^{o} B_{i}=r_{b}\left[\begin{array}{lll}
c \beta_{i} & s \beta_{i} & 0
\end{array}\right]^{T}, \beta_{i}=\frac{(i-1) 2 \pi}{5}, i=1,2,3,4,5
$$

where, $\beta$ represents the angle between $O B_{i}$ and the positive direction of $X$ axis,

The position vectors of points $A_{i}$ are expressed in reference coordinate frame as the following:

$$
{ }^{o} A_{i}=p+Q^{p} A_{i}
$$

where, position vector in the reference coordinate frame of the reference point $p$ for the platform is $\left[\begin{array}{lll}x & y & z\end{array}\right]^{T}, Q$ is the rotation matrix from the moving coordinate frame to the fix reference frame, which can be presented as.

$$
Q=\left[\begin{array}{ccc}
c \theta & s \psi s \theta & c \psi s \theta \\
0 & c \psi & -s \psi \\
-s \theta & s \psi c \theta & c \psi c \theta
\end{array}\right]
$$

For

$$
\left\|A_{i} B_{i}\right\|^{2}=\left\|{ }^{o} A_{i}-{ }^{o} B_{i}\right\|^{2}=l_{i}^{2}
$$

From the definition of the vector model, we can get the equation:

$$
l_{i}^{2}=\Phi(x, y, z, \psi, \theta)
$$

When the structure parameters $r_{a} 、 r_{b}$ are sure, if the pose 
parameters $(x, y, z, \psi, \theta)$ with respect to the platform are given, the driven chain lengths are obtained by the equation (6), that is, the inverse solution of kinematics position.

Equation (7) of the time derivative can be rewritten as matrix form

$$
B \dot{l}_{i}=A \dot{\varphi}
$$

where,

$$
\begin{aligned}
& B=\operatorname{diag}\left(2 l_{1}, 2 l_{2}, 2 l_{3}, 2 l_{4}, 2 l_{5}\right) \text {, } \\
& A=\left[\begin{array}{lllll}
\frac{\partial l_{i}}{\partial x} & \frac{\partial l_{i}}{\partial y} & \frac{\partial l_{i}}{\partial z} & \frac{\partial l_{i}}{\partial \psi} & \frac{\partial l_{i}}{\partial \theta}
\end{array}\right]
\end{aligned}
$$

The Jacobian matrix of the mechanism can be expressed as

$$
J=B^{-1} A
$$

Due to the linear velocity and angular velocity are different dimensions, the Jacobian matrix can be decomposed into

$$
J=\left[\begin{array}{ll}
J_{v} & J_{\omega}
\end{array}\right]
$$

\section{WORKSPACE ANALYSIS}

The workspace is the set of all points where the reference point of the moving platform can achieve in the space, which is the area of the robot manipulator, and is a pretty important index to measure the performance of the parallel manipulator.

Research on the workspace can be classified into two types, one is to search the position reachable workspace with constant orientation, the other is the dexterous workspace, owing to the middle branch restricts the rotation around $\mathrm{z}$ axis; the mechanism didn't have dexterous workspace. In this paper, we choose the constant orientation workspace. There are several methods, such as geometrical, analytical and numerical and so on, to analyze the workspace of the parallel robot manipulator. In this section, we research the workspace of the 5-SPS-UPU parallel manipulator based on the numerical method that is Monte Carlo simulation by the boundary search method.

It is particularly important to notice that, we should consider workspace during the design of the mechanism which determines the size of the parallel manipulator operating range actuator. And it is the foundation for the subsequent optimization. The workspace of parallel manipulator is mainly restricted by driven chains and constraint chain, as well angle constraints.

\section{A. Design Constraints Condition}

According to the structure characteristics, there are mainly two factors effect on its size and shape.

(1) The link length restriction: the link length is constrained by its structure and its constraint can be expressed by:

$$
l_{\min } \leq l_{i} \leq l_{\max }
$$

(2) Joint angel constraints: the links are generally attached to the moving platform by ball joints and to the fixed platform by ball joints which are equal to $\mathrm{U}$ joints. A ball joint is free to rotate about every axes, however, in practice, the moving platform is restricted by the physical construction of the geometry relation.
The angle, $\theta$, between the link and the z-axis of the fixed platform is used to the angle limitation of spherical joints, which can be described as(see Fig.2):

$$
\theta_{b i}=\arccos \left(\frac{l_{i} \cdot e b}{\left\|l_{i}\right\|}\right) \leq \theta_{s \max }
$$

Similarly, for the $\mathrm{S}$ joints in the moving platform the rotational angel is computed by:

$$
\theta_{a i}=\arccos \left(\frac{l_{i} \cdot e a}{\left\|l_{i}\right\|}\right) \leq \theta_{s \max }
$$

where, $e a, e b$ is the unit vector along the $z$ axis. $\theta_{s \max }$ is the maximum allowable rotational angels of the ball joints.

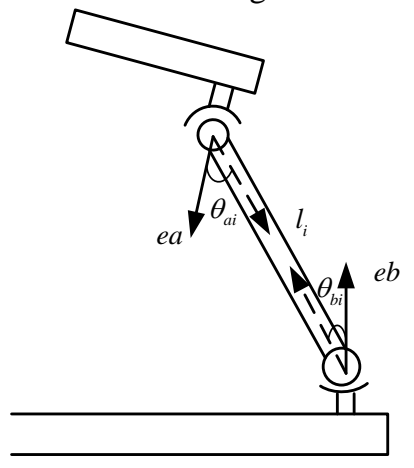

Fig. 2. Joint rotational constraints.

\section{B. The Search Process}

According to the above description, the research process of the workspace can be proceeded through the flow chart below (Fig. 3).

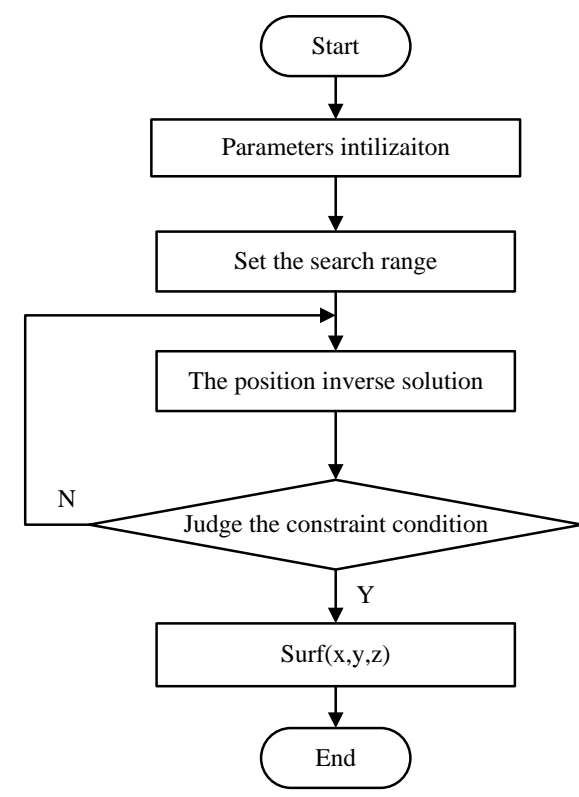

Fig. 3. Flow chart of searching workspace.

\section{NUMERICAL RESULTS OF THE WORKSPACE ANALYSIS}

For illustration of the workspace of the robot presented in this paper, the numerical solutions are calculated to analyze the characteristic of the robot. We set the parameters values of the mechanism are as follows (units: $\mathrm{mm}$ and radian):

$r_{a}=50, r_{b}=150, l_{\min }=50, l_{\max }=250, \theta_{s \max }=\theta_{u \max }=\frac{\pi}{4}$.

The search space of the workspace is $-200 \leq x \leq 200$, 
$-200 \leq y \leq 200,100 \leq z \leq 200$, the first case: $\psi=\theta=0^{\circ}$, the second case: $\psi=0^{\circ}, \theta=5^{\circ}$, the third case: $\psi=\theta=5^{\circ}$. This workspace of the parallel manipulator in different orientation are illustrated in Fig. 4, Fig. 5 and Fig. 6.

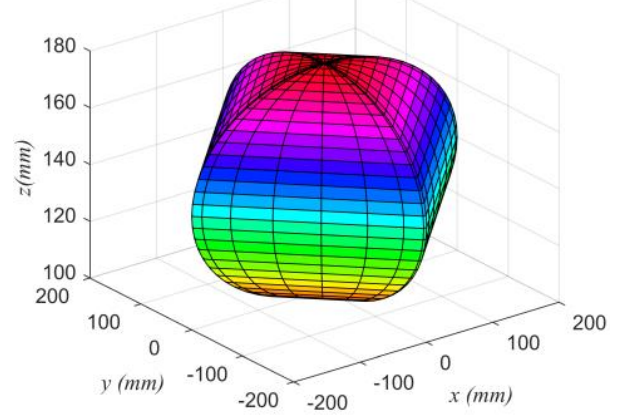

Fig. 4. Workspace of the parallel manipulator in orientation of $(0,0)$ degree.

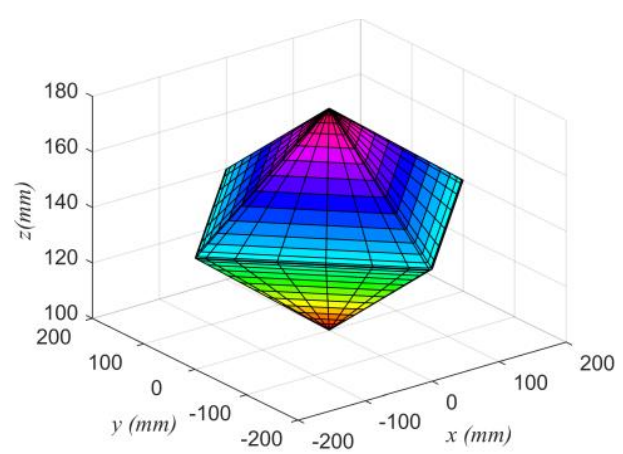

Fig. 5. Workspace of the parallel manipulator in orientation of $(0,5)$ degree

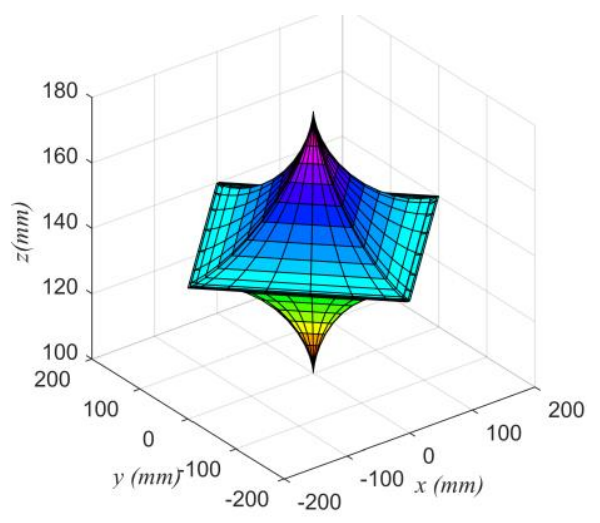

Fig. 6. Workspace of the parallel manipulator in orientation of $(5,5)$ degree.

The figures in different pose are sought by programming of the software MATLAB that is convenient and easy visualization of the calculation results. Form the figure above, we can see that the shape of the workspace is substantially similar to ellipsoid due to the limitation of the constraint conditions and the position workspace of the mechanism achieve largest when the roll angel $\psi=0^{\circ}$ and pitch angle $\theta=0^{\circ}$.

\section{THE DEXTERITY ANALYSIS AND THE CASES}

\section{A. Dexterity description}

When the parallel manipulator is close to a singular potion, the relation between output and input is highly-nonlinear. In order to describe the degree of movement distortion, kinematic dexterity index is introduced. For pure translation and rotation parallel manipulator, we can use the invers of the condition number of Jacobian matrix to describe the kinematic local dexterity, and the condition number of Jacobian matrix is used to measure the dexterity, which can be expressed as follows [18-19]:

$$
\mathbf{J}_{c}=\frac{\lambda_{M A X}}{\lambda_{M I N}}, 1 \leq \mathbf{J}_{c} \leq \infty
$$

where $\lambda_{M A X}$ and $\lambda_{\text {MIN }}$ are the maximum and minimum singular eigenvalues of matrix $\mathrm{J}$, respectively.

The condition number is in the range $1, \infty$, when the condition number reaches the minimum value 1 , the parallel manipulator is called kinematic isotropy, and it has the best kinematic transmission performance; when it close to infinity, the parallel manipulator is in the singular position.

\section{B. Case Analysis and Discussion}

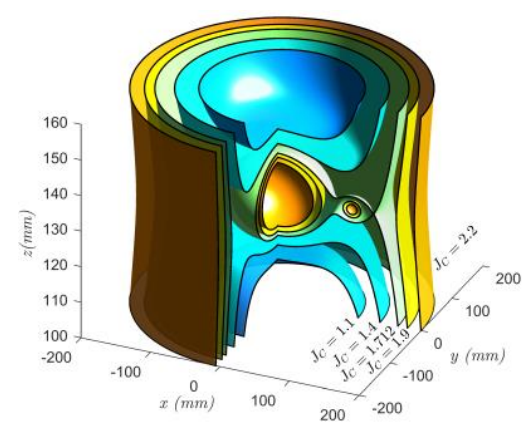

Fig. 7. Distribution of dexterity in different position.

Fig. 7 illustrates the distribution of the condition number under ideal workspace. Utilizing the results of calculation, the contour distribution of the condition number in the reachable workspace with consideration of the limit of the actuators stroke and rotational joint angle is evaluated and in Fig.7. From the Fig.7, we can see that the best kinematic dexterity is $J_{c}=1.1$. While the range of the kinematic dexterity is $(1.1,2.2)$. the workspace can be selected as the optimum task region. In the range of dexterity, with the position increasing, the condition number gradually raised, indicating the dexterity of the parallel manipulator is getting worse. However, in the search space, the range of the condition number varies between 1.2 and 2.2, and is far away from the ill condition region, indicating that the parallel manipulator has no singular configuration in the ideal workspace, and has good kinematic dexterity in the whole reachable workspace.

\section{CONCLUSIONS}

This paper develops a 5-DOF parallel manipulator design that aims to increase the workspace and dexterity of the mechanism. The main contributions of this paper are summarized as follows:

(1) A novel parallel robot manipulator 5-SPS-UPU which can generate three degrees of freedom translations and two degrees of freedom rotation was studied. The kinematic model and mathematical model were also investigated and the 
Jacobian matrix was deduced simultaneously.

(2) A general approach named the boundary search method is developed to seek the position workspace of the proposed parallel manipulator based on the inverse solution.

(3)The workspace of the parallel robot manipulator 5SPS-UPU was generated in this work, and the best kinematic dexterity is 1.1 , which not only has propitious basis for the application research design, but also is a good guide for the other similar parallel mechanism. For future works, a prototype will be manufactured and several relevant experiments will be conducted.

\section{ACKNOWLEDGMENT}

The authors would like to acknowledge the financial support of the Teaching Reform and Innovation Project for Shanxi Universities under Grant No.J2018136 and General Project of Changzhi Medical College under Grant QDZ201655. Furthermore, the work described in this paper is considerably revised and added some more information by our team. The author would like to express her gratitude to Ms. Fenxia Li who provides useful information in mechanical structure design and Ms. Chunhua Shi, Ms. Ping Li and Mr. Dianhua Bian who help us to collect the data in study area.

\section{REFERENCES}

[1] Y. Shneor, "Reconfigurable machine tool: CNC machine for milling, grinding and polishing," Procedia Manufacturing, 2018, vol. 21, pp. 221-227.

[2] A. A. Dyakonov, F. K. Nurkenov, and A. D. Kazansky, "Realization method of calculating the logarithmic decrement in turning and boring CNC machines technological systems," Procedia Engineering, 2017, vol. 206, pp. 1227-1231.

[3] G. Wang, H. Liu, and D. Yuan, "Force analysis of spatial 4-SPS/CU parallel mechanism," Transactions of the Chinese Society for Agricultural Machinery, 2012, vol. 43, no. 3, pp. 207-212+199.

[4] X. L. Chen, Q. Gao, and Y S. Zhao, "Dexterity measures of 4-UPS-UPU parallel coordinate measuring machine," Computer Integrated Manufacturing Systems, 2012, vol. 18, no. 6, pp. 1200-1208.

[5] D. Zhang, Global Stiffness Optimization of Parallel Robots Using Kinetostatic Performance Indices[M] Robot Manipulators Trends and Development, InTech, 2010.

[6] Y. Lu, "Computer simulation of a 3D free-form surface normal machining by 4SPS + RPS and 5SPS/UPU parallel machine tools,' International Journal of Computer Applications in Technology, 2007, vol. 30, 1/2, pp. 147-153.

[7] J. X. Fu and G. Feng, "Optimal design of a 3-leg 6-DOF parallel manipulator for a specific workspace," Chinese Journal of Mechanical Engineering, 2016, vol. 29, no. 4, pp. 659-668.

[8] F. L. Mura, P. Romanó, E. Fiore et al., "Workspace limiting strategy for 6 DOF force controlled PKMS manipulating high inertia objects,' 2018, vol. 7, no. 10, pp. 1-18.

[9] X. Fang, S. Zhang, Q. Xu et al., "Optimization of a crossbar parallel machine tool based on workspace and dexterity," Journal of
Mechanical Science and Technology, 2015, vol. 29, no. 8, pp. 3297-3307.

[10] G. Pond and J A. Carretero, "Formulating Jacobian matrices for the dexterity analysis of parallel manipulators," Mechanism and Machine Theory, 2006, vol. 41, no. 12, pp. 1505-1519.

[11] C. M. Gosselin and J. Angeles, "A global performance index for the kinematic optimization of robotic manipulators," Journal of Mechanical Design, 1991, vol. 113, no. 3, pp. 220-226.

[12] V. Rezania and S. Ebrahimi, "Dexterity characterization of the RPR parallel manipulator based on the local and global condition indices," Journal of Mechanical Science and Technology, 2017, vol. 31, no. 1, pp. 335-344.

[13] X. J. Liu, L. P. Wang, F. Xie et al., "Design of a three-axis articulated tool head with parallel kinematics achieving desired motion/force transmission characteristics," Journal of Manufacturing Science \& Engineering, 2010, vol. 132, no. 132, pp. 237-247.

[14] M. J. Tsai and H. W. Lee, "Generalized evaluation for the transmission performance of mechanisms," Mechanism and Machine Theory, 1994, vol. 29, no, 4, pp. 607-618.

[15] G. Wu and P. Zou, "Comparison of 3-DOF asymmetrical spherical parallel manipulators with respect to motion/force transmission and stiffness," Mechanism and Machine Theory, 2016, vol. 105, pp. 369-387.

[16] G. Cui, B. Wei, N. Wang et al., "Stiffness, workspace analysis and optimization for 3UPU parallel robot mechanism," Telkomnika Indonesian Journal of Electrical Engineering, 2013, vol. 11, no, 9, pp. 5253-5261.

[17] M. H. Saadatzi, M. T. Masouleh, and H D. Taghirad, "Workspace analysis of 5-PRUR parallel mechanisms," Pergamon Press, Inc. 2012.

[18] X. J. Liu, J. Wang, and H. J. Zheng, "Optimum design of the 5R symmetrical parallel manipulator with a surrounded and good-condition workspace," Robotics and Autonomous Systems, 2006, vol. 54, no. 3, pp. 221-233.

[19] Z. Shao, X. Tang, L .Wang et al., "Atlas based kinematic optimum design of the Stewart parallel manipulator," Chinese Journal of Mechanical Engineering, 2015, vol. 28, no. 1, pp. 20-28.

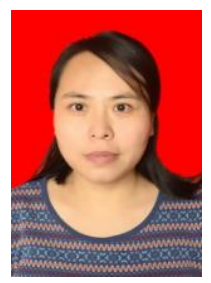

Ruixia Li was born in Qinshui, Shanxi, on June 26 1979. She received the B.E. degree in mechanical engineering from the Taiyuan University of Technology, Taiyuan, China, in 2001; the M.E. degree in mechanical and electronic engineering from the Taiyuan University of Technology, Taiyuan, China, in 2007.

She is currently an associate professor with the Department of Biomedicine Engineering, Changzhi Medical College, Shanxi. As the academic leader of rehabilitation equipment engineering, her current research interests include parallel mechanism, multi-objective optimization, control strategy research, rehabilitation robot and medical instruments.

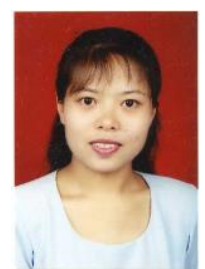

Fenxia Li received the B.E. degree from the Taiyuan University of Technology. Taiyuan, China, in 2000, the M.E. degree from the Taiyuan University of Technology. Taiyuan, China, in 2008.

She is currently a professor with the Department of NC Engineering, Shanxi Institute of Mechanical and Electrical Engineering. As a leader of the Huang danian's style teacher team, she takes the lead in innovation and solves technical problems. Her current research interests include NC processing, NC technology, mechanical processing. 